\title{
Liquid Crystal Functionalization of Electrospun Polymer Fibers
}

\author{
Dae Kyom Kim, Minsik Hwang, Jan P. F. Lagerwall \\ Department of Transdisciplinary Studies - Nanoscience \& Technology Program, Seoul National University, 443-270 Suwon, Korea, \\ and Advanced Institutes of Convergence Technology, 443-270, Suwon, Korea
}

Correspondence to: J. P. F. Lagerwall (E-mail: jan.lagerwall@Icsoftmatter.com)

Received 27 January 2013; revised 17 February 2013; accepted 19 February 2013; published online 29 March 2013

DOI: $10.1002 /$ polb.23285

ABSTRACT: A recently introduced new branch of applied polymer science is the production of highly functional and responsive fiber mats by means of electrospinning polymers that include liquid crystals. The liquid crystal, which provides the responsiveness, is most often contained inside fibers of core-sheath geometry, produced via coaxial electrospinning, but it may also be inherent to the polymer itself, for example, in case of liquid crystal elastomers. The first experiments served as proof of concept and to elucidate the basic behavior of the liquid crystal in the fibers, and the field is now ripe for more applied research targeting novel devices, in particular in the realm of wearable technology. In this perspective, we provide a bird's eye view of the current state of the art of liquid crystal electrospinning, as well as of some relevant recent developments in the general electrospinning and liquid crystal research areas, allowing us to sketch a picture of where this young research field and its applications may be heading in the next few years. (C) 2013 Wiley Periodicals, Inc. J. Polym. Sci., Part B: Polym. Phys. 2013, 51, 855-867

KEYWORDS: composites; core-sheath fibers; core-shell polymers; elastomers; electrospinning; fibers; functional responsive polymers; functionalization of polymers; liquid crystals; liquidcrystalline polymers (LCP); phase behavior; sensors
INTRODUCTION The strongly rising interest in electrospinning during recent years has several origins, the common factor being the potential of electrospinning for producing nano- to microscale diameter fibers of varying and often quite complex types. ${ }^{1-3}$ Already the basic technique provides a quite unique opportunity to produce extremely thin polymer fibers (diameter in the hundred nanometer range is easily achieved and thinner fibers can be produced with some more effort) of in principle unlimited length, using very small scale (in cost as well as size) equipment that can easily be installed in any physics or chemistry lab. The technique flourishes into its full beauty when two or more flow channels are combined in coaxial electrospinning, ${ }^{4}$ as it is then possible to include functional low molar mass liquids inside the fibers, which can be kept fully separated from the polymer sheath and-in case of multiple internal capillaries - from each other. Highly complex internal morphologies then become possible. ${ }^{5-7}$ In addition to presenting some interesting fundamental science research issues, coaxial electrospinning opens the door to the production of highly advanced composite fibers with entirely new application possibilities. The field displays a very stimulating degree of innovation, with new materials combinations being introduced at high rate. Among the functional materials that have now been electrospun together with the main polymer of the fiber are nanoparticles, organic light emitting diode components, organic photovoltaic and organic field effect transistor materials, laser gain media, liquid metals, live cells, and liquid crystals.
This perspective focuses on the latter category, discussing the benefits of combining the unique ordered fluids that liquid crystals constitute with polymer fibers. It also takes some of the other developments into account, as these advances can be of large importance for the opportunities to apply liquid crystal-functionalized electrospun fibers in devices. We begin by very briefly introducing liquid crystals to the readership outside their community, discussing also how they may respond to the very strong confinement inside a thin fiber. This is followed by a brief survey of the current state of the art of electrospinning fibers with liquid crystals. Then, some recent advances in coaxial electrospinning and in general liquid crystal research, with relevance from the perspective of applying liquid crystal-functionalized fibers, are concisely summarized. The perspective ends with an outline of some viable future directions and potential applications, where a particular focus is on the benefits of cross-fertilizing some of the different thrusts brought up in the previous discussion. In the interest of brevity, we assume that the reader is acquainted with electrospinning, referring newcomers to this field to some of the many excellent reviews on the topic, for example, Refs. 1-3.

\section{LIQUID CRYSTALS AND THEIR RESPONSE TO CONFINEMENT}

A liquid crystal combines fluidity with long-range order., ${ }^{8,9}$ In the simplest case, the nematic phase $(\mathrm{N})$, the order is purely orientational, that is, the molecules (typically rod- or 
Jan Lagerwall got his M.Sc. in Physics and Ph.D. in Materials Science at Chalmers University of Technology, Sweden, working primarily with chiral smectic liquid crystals. During post-docs in the USA and Germany, he started working also with nanoparticles and lyotropics, and he developed the new field of liquid crystal electrospinning. After three years as group leader at Martin-Luther University Halle-Wittenberg, Germany, he took on a professorship at Seoul National University, Korea, in 2010. He holds the Swedish docent title in Physics and the German Habilitation in Physical Chemistry. Current research interests range from liquid crystal phase transitions in spherical shells to wearable technology, via polymer physical chemistry and nanocrystalline cellulose self-assembly.

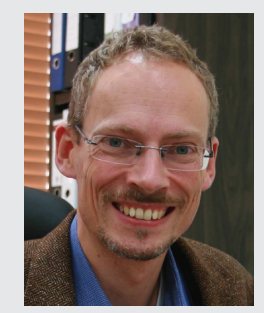

Dae Kyom Kim got his M.S. degree in polymer science at Han Nam University, Daejeon, Korea in 2002. During his M.S. course, he worked with synthesis and characterization of photosensitive polymers for semiconductor applications. After six years, as researcher at Samsung electronics, developing photolithography processes and materials, he then joined Prof. Lagerwall's group as Ph.D. student in 2012. His current research interest is fabrication of liquid crystal functionalized fiber mats for wearable technology applications via electrospinning.

Minsik Hwang was born in Seoul, Korea. Currently, he follows a combined master and doctorate program at the Graduate School of Convergence Science and Technology, Seoul National University, where he is now a master student in Prof. Lagerwall's group. His research focuses on liquid crystal-polymer composites fabricated by electrospinning for making smart textiles.

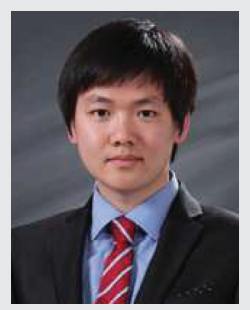

disc-shaped) tend to align along the same direction (indicated by the director, $\mathbf{n}$ ), compare Figure 1(a). In smectic and columnar phases [Fig. 1(b-d)] partial positional order occurs additionally, in one and two-dimensions, respectively. Liquid crystals are formed by some molecules on their own, typically combining a rigid core with flexible end chains to stabilize the partially ordered state. Such liquid crystals are referred to as thermotropic, indicating that temperature is the main thermodynamic control variable. Lyotropic liquid crystals, on the other hand, form only in the presence of a solvent, and here the concentration of the solute or of dispersed particles typically determines which phase is formed. In electrospinning work until now, only thermotropics have been incorporated, although nothing prevents lyotropics per se to be used as well.

A highly useful aspect of liquid crystals is their strong response function, that is, they give a strong macroscopic response to a small external influence. ${ }^{8,9}$ The technologically most important example is the response to electric fields of thermotropic nematics, which constitutes the basis for their immensely successful application in flat panel displays, but many other examples exist. A striking phenomenon is for instance the strong sensitivity to chirality. Many liquid crystal phases develop a helical superstructure, some even ferro- or antiferroelectric properties, as a response to chiral dopants. The helix pitch can be on the order of visible light wavelengths, then giving rise to a photonic bandgap and selective reflection, that is, strong iridescent color arising from Bragg reflection of light, as shown in Figure 2. Combined with the ease in tuning the pitch and thereby the band gap by temperature, electric fields or mechanical stress, this has inspired numerous groups to use chiral liquid crystals for instance as soft matrices for mirrorless lasing. ${ }^{10,11}$ Most often the chiral nematic phase $\left(\mathrm{N}^{*}\right)$ is used, frequently referred to as cholesteric in recognition of its original observation in a cholesterol derivative.

A fascinating material arises if a reactive liquid crystal is polymerized and lightly crosslinked, to form a liquid crystalline rubber. This can show quite extraordinary mechanical response to temperature changes or to illumination. ${ }^{12,13}$ The reason is that the presence or absence of long-range order strongly influences the polymer chain conformation and thereby the shape in this liquid crystal elastomer. In the liquid crystal phase, the polymer chains adopt an anisotropic conformation but in the isotropic phase a regular isotropic coil prevails. Due to the crosslinks, this conformational change couples to a macroscopic shape change and the liquid crystal elastomer can thus function as an actuator triggered by whatever external influence that can induce the phase transition between nematic and isotropic. 


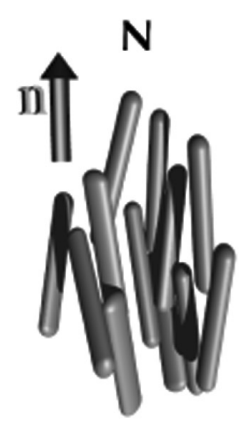

a

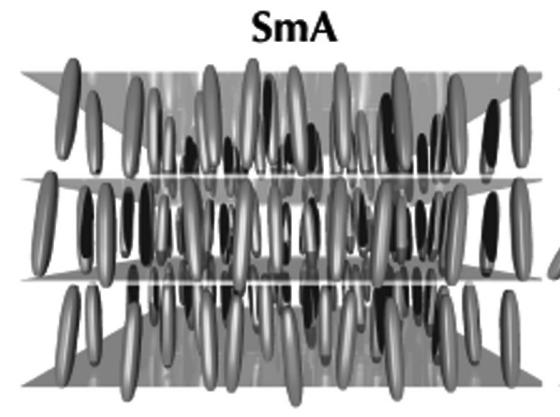

b

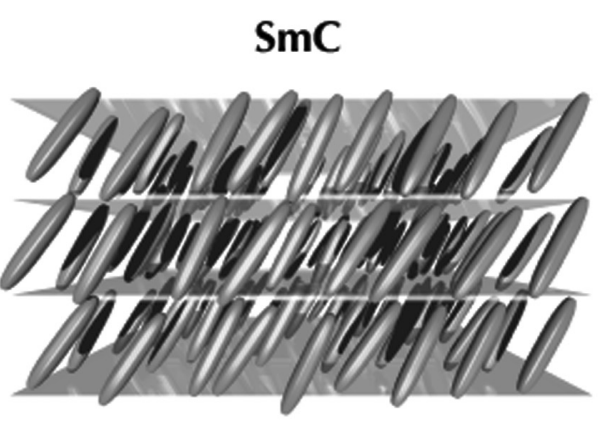

c
Col.

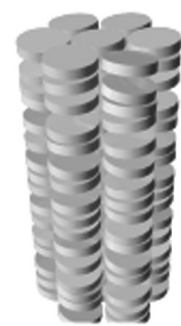

d

FIGURE 1 Cartoon illustrations of the three most important liquid crystal phases from rod-shaped molecules, nematic (a), smectic-A (b) and smectic-C (c), and the columnar phase formed by disc-shaped molecules (d). The director ( $\mathbf{n}$ ) is defined as an indicator of the average orientation of the molecule symmetry axis using the nematic as example. The layering in the smectic phases has been exaggerated and complemented with imaginary separating planes for clarity. In reality, the smectic order is much more subtle.

The attractive self-assembled ordered structures and the immense response function are the prime applied reasons for functionalizing electrospun fiber mats with liquid crystals. The optical properties of an incorporated liquid crystal can be transferred to the mat as a whole ${ }^{14}$ and structural rearrangements in the liquid crystal will typically affect the macroscopic appearance. With liquid crystal elastomer fibers strong actuation can be expected and a number of other attractive liquid crystal functions can potentially be put to good use in the fibers. There is also a fundamental scientific interest in studying the liquid crystal inside the fibers, primarily related to the effects from the strong encapsulation inside the narrow fiber cores on the phase sequence or on the development of ordered superstructures. Liquid crystals can adapt in diverse ways to confinement, the response ranging from helix unwinding to the complete disappearance of certain phases. ${ }^{15}$ When encapsulated inside electrospun fibers, strong effects on the phase sequence have been found for some liquid crystals, ${ }^{14,16}$ whereas for others the effect was less dramatic. ${ }^{17}$ Moreover, an interesting quantization of the helix pitch was found in case of cholesteric cores, ${ }^{14}$ as discussed further below.

\section{THE STATE OF THE ART IN RESEARCH ON LIQUID CRYSTAL-FUNCTIONALIZED ELECTROSPUN FIBERS}

The first successful attempt to electrospin fibers with liquid crystal inside was reported by Lagerwall et al. in $2008 .{ }^{16} \mathrm{~A}$ room temperature nematic mixture was spun as a core inside a composite sheath consisting of polyvinylpyrrolidone (PVP)
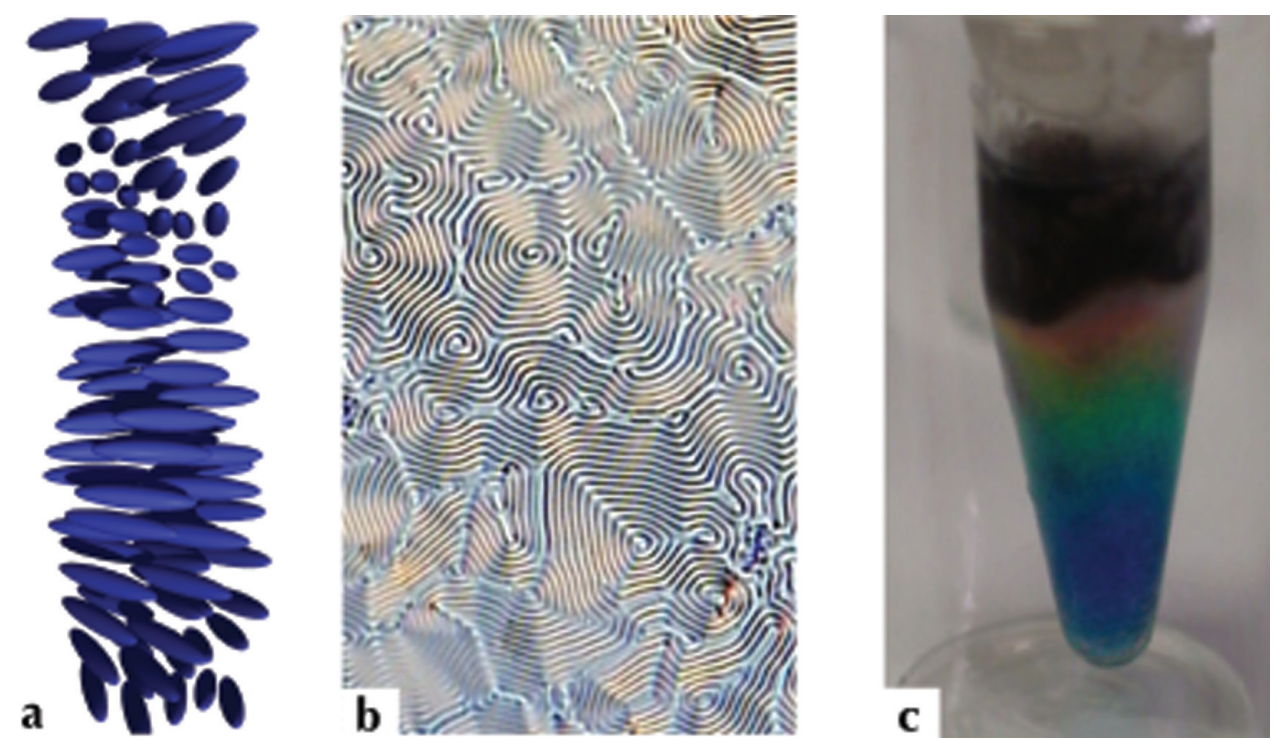

FIGURE 2 The helical modulation of the director in a cholesteric liquid crystal (a) gives rise to characteristic textures with fingerprint-like patterns (b) for helix period (pitch) on the order of microns. In case the pitch is shorter, reaching the order of visible light wavelengths, the cholesteric becomes a self-assembled photonic crystal that displays spectacular colorful reflection (c). Because the pitch is frequently highly sensitive to temperature, the color can vary over the whole spectrum in case of a temperature gradient as in this photo (top is colder than bottom). 


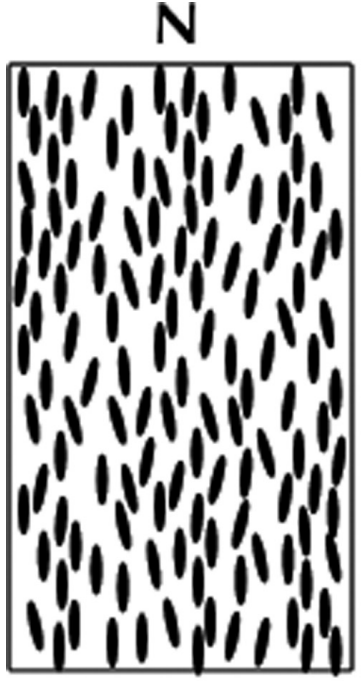

a
SmA

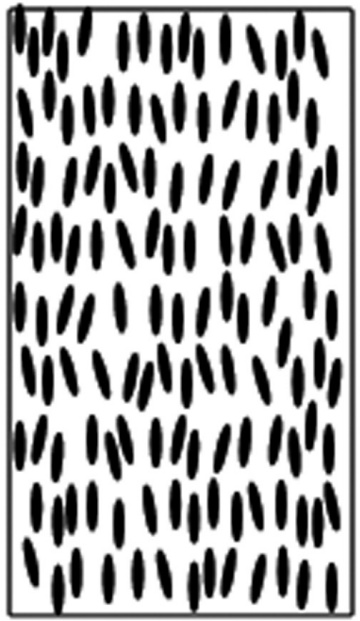

b

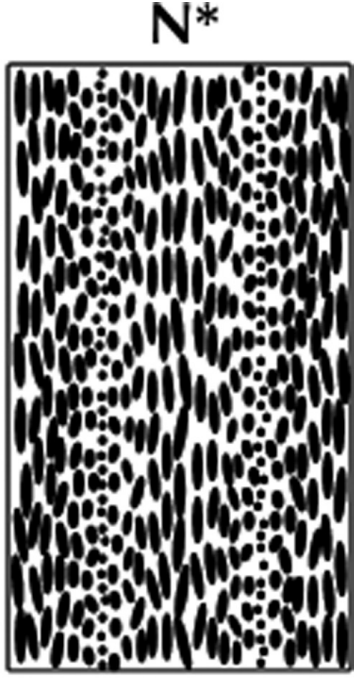

$\mathbf{C}$

FIGURE 3 Illustrations of how nematic (a), SmA (b), and cholesteric (c) phases align within electrospun fibers. The drawings illustrate the molecular arrangement in a cross section at the mid plane of the fiber. The ellipsoids correspond to the projection of the molecules on this plane, thus a shortening of the ellipsoids as in (c) corresponds to a rotation of the molecules into the viewing direction.

and $\mathrm{TiO}_{2}$. Polarized Raman spectroscopy gave a clear signature of alignment of $\mathbf{n}$ primarily along the fiber. This molecule arrangement is illustrated in Figure 3(a). A surprising observation was that the orientational order persisted about $20 \mathrm{~K}$ beyond the bulk clearing point of the liquid crystal mixture on heating, and the disappearance of the order was continuous until the end. This means that the encapsulation of this mixture inside the fiber dramatically stabilized the nematic phase and gave an apparent change from abrupt to continuous phase transition. The latter is most likely an effect of surfaceinduced order close to the sheath coexisting with isotropic phase growing from the very center of the core.

Enz et al. ${ }^{14,17}$ later succeeded in spinning fibers without any inorganic component, with nematic as well as smectic phases inside a pure PVP sheath. The absence of $\mathrm{TiO}_{2}$ had the important advantage that the liquid crystal core could now easily be investigated by optical microscopy, as the pure PVP sheath is transparent, gives very little scattering on its own and has a weak birefringence, substantially lower than that of the enclosed liquid crystal (PVP is an amorphous polymer, hence birefringence arises only from slight stretching-induced chain alignment retained in the glassy state). The optical investigations of fibers containing octadecylcanobiphenyl (8CB; a commonly studied single-component liquid crystal with a smectic-A (SmA) phase at room temperature and a nematic phase at slightly elevated temperature] confirmed that also in this case the director aligned along the fiber. This alignment, most likely resulting from the strong planar alignment dictated by the polymer wall, means that the smectic layers have exceptionally small lateral extension, equal only to the cross section of the internal fiber channel [Fig. 3(b)].

Even more interesting was the study on a short-pitch cholesteric liquid crystal spun inside PVP fibers. ${ }^{14}$ The planar director alignment at the interface to the polymer now ensured an orientation of the cholesteric helix perpendicular to the fiber [Fig. 3(c)], meaning that the selective reflection arising from the photonic crystal properties of cholesterics along the helix axis is seen when observing the fibers perpendicular to the fiber axis, for example, lying down on a microscope slide as in Figure 4. An attractive and at first surprising aspect of these fibers is that all colors can be observed, although the color of the bulk liquid crystal is very well defined (a greenish blue in this particular case). The color variation becomes understandable when considering the constraints imposed by the cylindrical symmetry of the fiber on the development of the helical director arrangement. Because $\mathbf{n}$ must be parallel to the

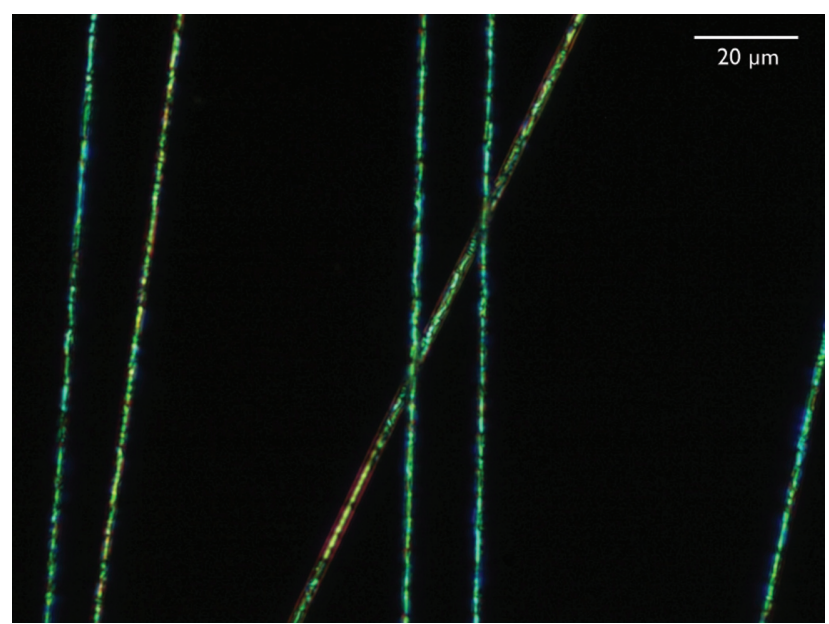

FIGURE 4 Coaxially spun fibers with PVP sheath and a short-pitch cholesteric liquid crystal core. Note the variations in reflection color, resulting from slight variations in core diameter, imposing slightly different director helix pitch and thus shifts in the photonic band gap. ${ }^{14}$ 
fiber at the boundary as well as at the center, the helix pitch, and consequently the color, gets strongly quantized (this is explained in detail in Ref. 14). The fundamental effect is wellknown from wedge cells, where it is referred to as the Cano effect, ${ }^{8}$ but the cylindrical geometry makes the quantization twice as strong and the very small diameter of the electrospun fiber further renders the range of colors quite spectacular. An attractive consequence is that the color thereby directly reflects the diameter of the fiber core, hence variations in the diameter can be detected simply by optical inspection.

Coaxial electrospinning is not the only way of achieving liquid crystal-filled fibers. An interesting alternative approach using a single-fluid spinneret was introduced by Buyuktanir et al. ${ }^{18}$ They spun a mixture of a liquid crystal and polymer (polylactide) in a common solvent mixture, obtaining the coaxial fiber geometry via phase separation as the solvent evaporated during spinning. The coaxial spinneret is generally preferable in terms of controlling the fiber geometry and ensuring a welldefined coaxial fiber, however. There is also a risk of complete phase separation occurring when using a simple spinneret, that is, liquid crystal droplets separate entirely from the polymer fiber. Indeed, the fibers reported in Ref. 18 show many inhomogeneities, but it is nevertheless impressive that this simple approach can be used.

Rather than electrospinning a low-molar mass liquid crystal inside a polymer sheath it can be of interest to spin a liquid crystalline polymer, although the molar mass typically available for such polymers may not always be sufficient for supporting electrospinning. Two reports following this strategy exist in the literature. ${ }^{19,20}$ Krause et al. ${ }^{20}$ electrospun a photocrosslinkable main-chain liquid crystalline polymer dissolved in chloroform, exposing the jet with strong UV light to produce nematic elastomeric fibers in situ during spinning. The authors reported good director alignment along the fiber, as confirmed by polarizing microscopy. Surprisingly, they did not discuss the phase transition-induced actuation properties of the fibers, possibly an indication that these were poorer than expected. If so, one may speculate that actuation could have been inhibited by attachment of the fibers on the collector substrate, as well as by a random network morphology. If one can spin free-hanging and uniformly oriented fibers, a substantial actuation should be expected, as seen in liquid crystal elastomer fibers produced by wet spinning from a solution of a photocrosslinkable SmA main-chain polymer. ${ }^{21}$ Smectic main-chain liquid crystal polymer fibers were the topic also of the electrospinning study of Nakashima et al. ${ }^{19}$ but these fibers were not crosslinked, hence they cannot be expected to actuate.

\section{OPENINGS FOR CROSS-FERTILIZATION}

The results described above provide a fundamental proof of concept that liquid crystals can be well-incorporated into fibers produced by electrospinning, in diverse forms and following varying procedures. However, they only constitute the birth of the field. The future development can be expected to include important practical improvements, allowing the production of new composite functional fibers with unique application potential in innovative devices. To this end, a number of recent achievements in the general electrospinning field as well as some current trends of liquid crystal research may come to play an important role, the most important ones summarized in the following.

\section{Recent Key Advances in Electrospinning}

Considering the benefits of the coaxial electrospinning technique for introducing liquid crystals into the fibers, some recent innovations in the design of the coaxial spinneret are of great interest. With regular coaxial electrospinning, the liquid crystal can be encapsulated inside the polymer but in practice there is only one surface bounding the liquid crystal. This makes it difficult to interact via electric fields with the liquid crystal. Because of the strong response of the liquid crystal to electric fields and the resulting potential to realize for example, simple display-like fibers, this is a worthwhile goal. A convenient way to solve this problem is to raise the complexity of the spinneret one level by introducing a third coaxial capillary. Although this has not yet been done with liquid crystals, Chen et al. achieved this type of triple-coaxial fiber configuration using paraffin oil as intermediate phase, ${ }^{5}$ compare Figure 5(a). The innermost as well as the outermost phases were polymer solutions, thus giving a solid polymer central core as well as a solid polymer outer sheath separated by the cylindrical layer of paraffin oil after evaporation of the solvent.

If the innermost and outermost phases can be made electrically conductive, we would have provided the fibers with cylindrical coaxial electrodes allowing a convenient means of applying an electric field over the intermediate layer, which then should be a liquid crystal rather than paraffin oil. Different approaches can be considered for achieving the conductive core and sheath. First, both polymer phases, or only the sheath solution, can be doped with conductive nanoparticles like carbon nanotubes (CNTs), making the solid polymer conductive if the CNTs are present at a concentration above percolation threshold. A difficulty in this approach is that a good surfactant normally must be used for dispersing the nanotubes and preventing aggregation, but its presence has a highly negative effect in terms of raising the contact resistance between the tubes. This lowers the conductivity of the composite and probably also raises the percolation threshold, requiring a higher concentration of CNTs in the polymer. However, a recently introduced new method to disperse the nanotubes below the Krafft temperature of the surfactant to achieve an absolute minimum of its concentration ${ }^{22}$ holds promise for minimizing this negative effect, thus achieving high-performing electrically conductive CNT-polymer composites.

Second, for the core electrode, a very interesting alternative is to use metal alloys that are liquid at room temperature, such as GalInStan (a eutectic alloy of gallium, indium, and tin), as the core fluid in the electrospinning experiment. This gives a core electrode with excellent conductivity and perfect compatibility with wearable electronics due to its liquid state. Although the very high surface tension of GalInStan (it has been reported to be more than $530 \mathrm{mN} / \mathrm{m},{ }^{23}$ an order of magnitude greater than 

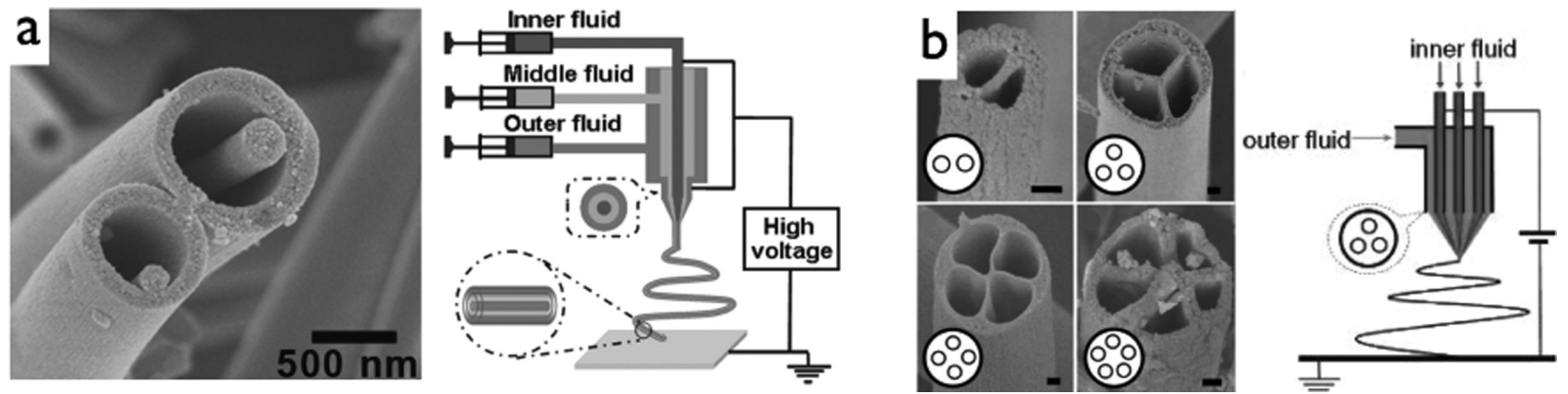

FIGURE 5 Examples of more advanced internal cross-sections possible in electrospun fibers, by using a triple coaxial spinneret (a) or multiple bundled internal capillaries (b). Adapted from (a) Ref. 5, [with permission from copyright (2010) American Chemical Society] and (b) Ref. 7 [with permission from copyright (2007) American Chemical Society].

the surface tension of water) would seem to suggest that this would be a very difficult-if not impossible-liquid to work with in electrospinning, a recent report in fact described just this: Yang et al. ${ }^{24}$ claim to have succeeded in electrospinning organic light-emitting diode (OLED) fibers using a GalInStan core electrode. An important reason for the success in this venture is probably the very rapid oxide skin formation when GalInStan is exposed to oxygen, giving the liquid metal gel-like properties and stabilizing highly nonspherical morphologies. ${ }^{23}$ It also leads to a tremendous contact angle hysteresis, giving the false impression that GalInStan wets almost any surface. This skin should thus facilitate the large-area contact between the liquid metal and the surrounding polymer solution during spinning. It should also help to suppress the Rayleigh instability and thus counteract the separation of the GalInStan core into droplets. Although there are several important unresolved issues to investigate here regarding the behavior of the liquid metal and its interaction with the surrounding polymer solution, the report that electrospinning with liquid metal core is possible is very promising for future advanced fiber devices. If it can be reliably used with varying polymer solutions, it would provide an excellent approach to introduce fully flexible, stretchable, and durable electrodes.

Another highly interesting development in coaxial spinneret design is to introduce multiple bundled internal capillaries. ${ }^{6,7}$ This results in a multicore fiber where each internal channel can be well-sealed from the adjacent ones if the spinning conditions are optimized, see Figure 5(b). By changing the number of inner capillaries Jiang and coworkers succeeded in spinning fibers with twofold, threefold, fourfold and fivefold symmetry of the internal morphology, thus with the same numbers of well-separated internal channels. Although the reports so far are only for identical or very similar core liquids (paraffin oil was used in all capillaries in Ref. 7 whereas the two capillaries in Ref. 6 were pumping hexadecane and icosane, respectively) truly interesting composite fibers will arise when the different capillaries introduce different liquids with complementary properties, for example, liquid crystal in one capillary and GalInStan in another. The future will tell if it will be possible to retain the continuity of all channels also in these cases where each inner fluid may have very different surface tension and extensional viscosity. To some extent, the differences can be compensated for by using different pump pressures and capillary diameters for the different core fluids, and most likely surfactants or similar additives must be used to reduce the interfacial tension. If successful, this can certainly constitute a very important breakthrough for composite fiber electrospinning.

With the extraordinary optical properties and the versatile means of modulating them dynamically that liquid crystals offer, one may contemplate the use of liquid crystalfunctionalized electrospun fibers as active optical fiber components. In particular for integrated optical nanodevices, the fibers might constitute attractive and useful nanophotonic components. The realization can be practically challenging, however, considering that the diameter of electrospun fibers is typically a few orders of magnitude lower than that of most optical fibers. Coupling of the light into or out of the electrospun fibers thus becomes a difficult task. Nevertheless, one can in fact find works demonstrating the potential of standard coaxial electrospun fibers (without liquid crystal) as (passive) optical fibers. Kwak et al. ${ }^{25}$ used a smart trick to avoid the need to couple light into the fibers, namely by having secondary light being emitted inside the fiber. They spun coaxial fibers with polycarbonate core and polymethylmethacrylate sheath (a combination that fulfills the requirement for optical fibers of higher refractive index in the core than in the cladding), with the commonly used laser dye DCM (4-(dicyanomethylene)2-methyl-6-( $p$-dimethylaminostyl)-4H-pyran)) mixed into the core. In this way, they could excite fluorescent emission from the DCM within the fiber core by illuminating the whole fiber mat with a UV lamp, yielding clear evidence of light being guided along the fiber, compare Figure 6.

A further important recent development regards the sheath polymer. So far, most liquid crystal electrospinning work has been done using PVP as sheath polymer, sometimes with added $\mathrm{TiO}_{2}$ as inorganic component. This is convenient because PVP can be obtained with very high molar mass and is easy to spin from an ethanol solution, which renders it immiscible with the thermotropic liquid crystal core fluid. The problem is that PVP is highly hygroscopic, inelastic, and rather soft, giving it poor mechanical properties. Thus, for future development alternative sheath polymers should be identified, 


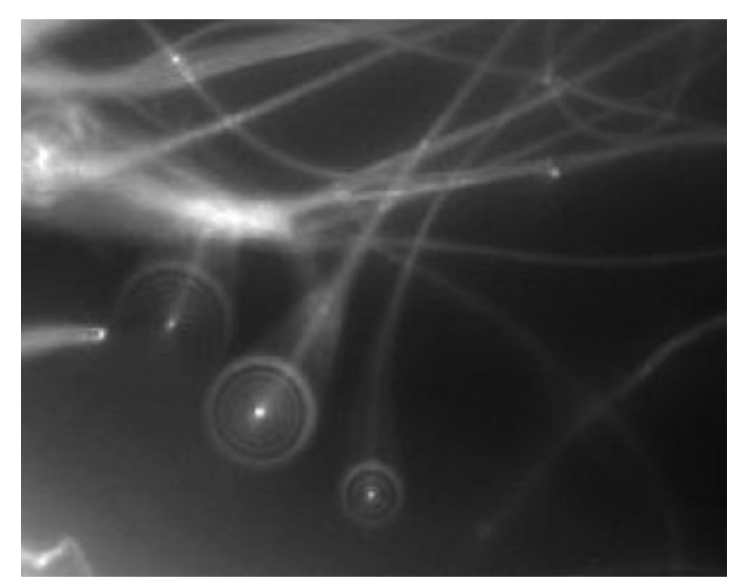

FIGURE 6 Fluorescence image demonstrating light guiding in coaxial polycarbonate-polymethylmethacrylate fibers with incorporated laser dye acting as a fiber-integrated secondary light source. Reproduced from Ref. 25, [with permission from copyright 2008 WILEY-VCH Verlag GmbH \& Co. KGaA, Weinheim].

preferably compatible with textile applications yet retaining immiscibility with the liquid crystal core and providing easy electrospinnability.

A promising approach seems to be to use polyvinylalcohol (PVA) as sheath polymer, as the hydroxyl pendant groups provide a convenient handle for crosslinking the polymer. Once crosslinked, PVA becomes water resistant and the fibers then provide the basis for durable and strong fabrics (commercially known as vinylon). A number of groups have developed routes to produce crosslinked PVA fibers by electrospinning, where the crosslinking stage can be performed either in situ ${ }^{26}$ or after electrospinning. ${ }^{27}$ Although so far none of these studies dealt with coaxial fibers, it should be relatively straightforward to extend these approaches to work with an encapsulated inner fluid. However, the dynamically changing physical properties of the solution during in situ crosslinking may make it more challenging to find conditions where outer and inner solutions are well-matched. The strategies involving crosslinking after spinning are therefore likely to be more successful. Another alternative is to spin the sheath out of for example, polyamide dissolved in formic acid, ${ }^{28}$ although it is not certain if the solubility of the liquid crystal in formic acid is sufficiently low to ensure retained stable coaxial geometry.

For some applications of electrospun fibers, a porous sheath can be highly advantageous, for example, in sensing applications or when the absolute maximum in surface area is called for. Two approaches have been introduced to achieve this with some degree of control of the porosity. The Rabolt group found that electrospinning in a humid atmosphere can give rise to a high density of very small pores, resulting from water droplets condensing on the fiber surface (cold due to the rapid evaporation of the solvent) while this is still in a liquid state. ${ }^{29}$ When the fiber solidifies shortly afterwards, the water droplets are still present, producing the porous indentations which then remain permanently after the evaporation of the water. An interesting alternative introduced by the Xia group is to spin the fibers into a cryogenic bath of for example, liquid nitrogen. ${ }^{30}$ This leads to thermally induced phase separation between regions rich and poor in solvent, respectively, finally yielding a porous morphology after all solvent has been evaporated in vacuo. It is important that the solvent stays frozen until the vacuum drying, during which the solvent thus sublimes in this procedure, in many ways reminiscent of freeze drying. As solvent is distributed throughout the polymer when the fibers are quench-frozen in the cryogenic liquid, pores are generated throughout the polymer when following this procedure, in contrast to the surface-porous fibers produced following the Rabolt approach. So far neither technique has been applied to coaxial fibers but there is no apparent reason why this would not be possible.

\section{Recent Trends in Liquid Crystal Research Ripe for Introducing Electrospinning}

Apart from the afore mentioned advances in general electrospinning, a number of exciting new trends in liquid crystal research have also appeared during the last years. So far, they have no link to electrospinning but there is a clear potential in combining them with this new approach for making interesting liquid crystal-polymer composites. Here, we will briefly introduce three of these new research topics but the list could easily be extended, as liquid crystal research is presently going through a very creative phase with many new subfields regularly being introduced. ${ }^{9}$

\section{High-Specificity Gas Sensing Using Liquid Crystals with Tailored Dopants}

The concept of using cholesteric liquid crystals for sensing the presence of organic vapors, relying on the increase or decrease in pitch and consequent change in color on exposure to the analyte for the sensing mechanism, was developed in the $1990 \mathrm{~s}^{31}$ and good quantitative performance was demonstrated. A critical problem that hampered the further development, however, was the lack of specificity: any vapor that dissolves in the liquid crystal will have some effect on the helix pitch. Although the situation could be improved somewhat through an elaborate analysis of the response, ${ }^{31}$ this makes the method much more awkward to use and in a sense negates the benefit of using cholesteric liquid crystals as sensors.

A major breakthrough that addresses the selectivity issue was recently reported by Han et al. ${ }^{32}$ By using chiral dopants that were tailor-designed for sensitivity to specific analytes (here $\mathrm{O}_{2}$ and $\mathrm{CO}_{2}$, respectively), responding by a conformational change that changes the helical twisting power of the dopant, they succeeded in producing sensors useful in for example, food packaging. Exposure to the analyte resulted in a distinct color change from green to red or from green to yellow. By introducing other appropriately designed chiral dopants, the concept should become available for a large range of analytes, including biologically active substances. The concept is attractive in many respects, because it allows for a gas or biosensor that operates at room temperature, with no need for a power supply, and with signal directly detectable by the 

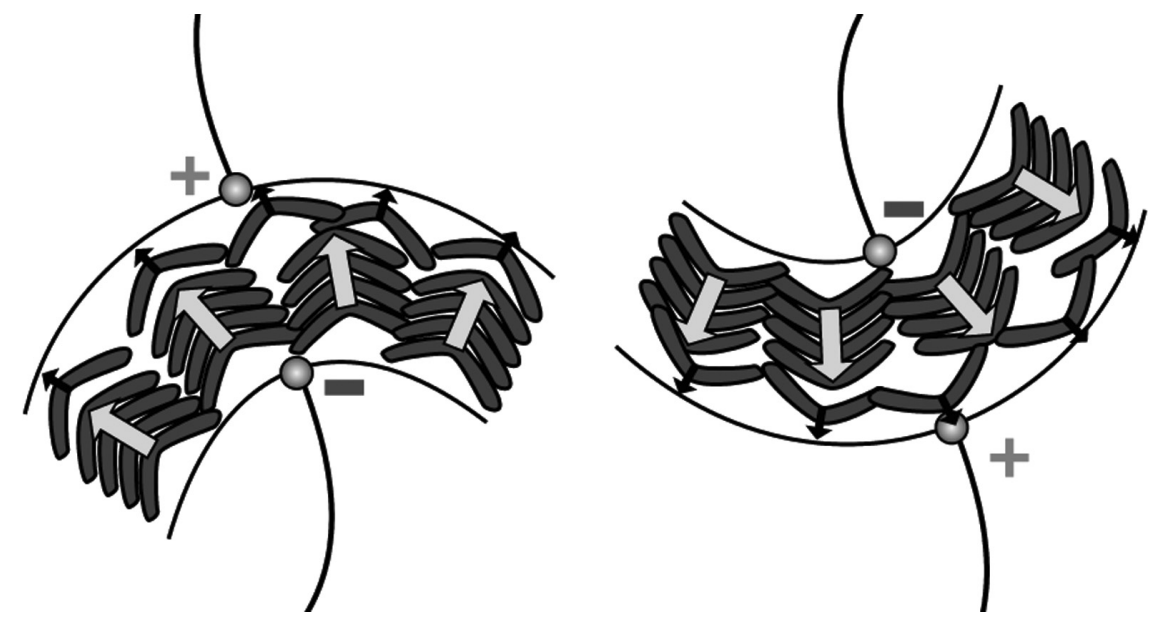

FIGURE 7 Cartoon illustrating the piezoelectric response of bent-core nematic liquid crystals, forming small smectic-like clusters within the phase, with uniform electric polarization (indicated by arrows). When the macroscopic sample, for instance, a fiber containing the liquid crystal, bends, the molecules tend to bend in the same direction to optimize the packing, leading to a macroscopic surface charge that reverses on flexing up and down.

naked eye, even by an untrained observer. The packaging of the sensor could, however, still be improved. In the work by Han et al., the cholesteric was coated as a film on top of a triacetylcellulose foil as a carrier, leaving the liquid crystal inaccessible to the analyte from the bottom and unprotected from the top. It could thus easily be smeared off, contaminated, or otherwise damaged during handling of the sensor. For large-scale production and distribution of sensor devices, it is clear that an appropriate encapsulation, compatible with the sensing function, would be necessary.

\section{Mechanical Sensors Utilizing Cholesteric Liquid Crystal Elastomers}

The striking colors of cholesterics and their sensitive response to various types of influence have been used also in other types of sensors. An attractive approach to making mechanical sensors, reacting to pressure or tensile stress, is to polymerize a cholesteric into an elastomeric sample. The Finkelmann group used this concept for a mirrorless dye laser, using the cholesteric elastomer as a periodic amplification medium that allows tuning of the laser wave length by stretching. ${ }^{11}$ Seeboth et al. $^{33}$ used the same phenomenon to make a piezochromic sensor, responding to pressure by a change in color.

In both these cases, the response takes advantage of the direct coupling between the liquid crystalline order and the shape and size of the macroscopic elastomer sample. Attractive aspects are for example, a fast response, full reversibility and, again, no need for electrical power when using the system as a sensor. Because the elastomer is made by crosslinking the liquid crystal (typically UV-initiated) in its normal sample configuration, the concept is compatible essentially with any sample shape, including fibers.

\section{Electromechanical Power Conversion Based on Liquid Crystals with Giant Flexoelectric coefficients}

The flexoelectric effect has been studied in liquid crystals since the 1970 s. $^{34}$ It is an analog to piezoelectricity, where an electric polarization, and thus a surface charge, appears as a result of a coupling between a flexing-induced distortion of the long-range orientational order, an asymmetric molecule shape, and a nonzero molecular dipole moment. Generally, the effect is rather weak and the main interest has been in the context of the flexoelectrooptic effect, ${ }^{34,35}$ in which the birefringent optical properties respond very rapidly to an applied electric field due to the flexoelectric coupling.

A few years ago, the situation changed dramatically as the newly discovered nematic liquid crystal phases from bent-core mesogens turned out to exhibit giant flexoelectric coefficients. ${ }^{36}$ These were so large that a new style of operation becomes of interest, that is, where the surface charge generated by director field distortion is picked up by electrodes in contact with the sample. Repeated back-and-forth flexing then yields an AC current, enabling the bent-core nematic to be used as a mechanoelectrical energy converter competitive with devices built on solid-state piezoelectrics. In fact, under practical application conditions, liquid crystals outperform many inorganic or polymer piezoelectrics, ${ }^{37}$ as one must take the maximum strain gradient into account (which is substantially higher for liquid crystals) and as liquid crystal-based composites can be made very thin, ensuring high efficiency.

This new concept was pioneered by Jakli and coworkers ${ }^{37}$ who combined bent-core nematics and elastomers to provide sufficient physical support while at the same time ensuring flexibility and strong coupling between macroscopic sample flexing and generation of surface charge. It turns out that bent-core nematics contain ferroelectric and thus spontaneously polarized smectic-like nanoclusters. When the sample is flexed, the molecules will pack more efficiently if their molecular bend direction matches the flexing direction of the sample, compare the schematic illustration in Figure 7. This will force the polarization of the clusters to flip on reversing the flexing direction, thus leading to alternating charge 
separation on the opposite sides of the sample which is the basis for the generator concept.

Because only the surface charge contributes to the function, the efficiency can be expected to be greater the larger the surface-to-volume ratio of the sample. So far, the samples were prepared as thin films with one electrode on each side, but there is still good potential for scaling down to increase the efficiency. By replacing the films with thin fibers, the surface-to-volume ratio increases dramatically, hence electrode-equipped electrospun fibers containing bent-core nematics could provide outstanding performance from this point of view. Moreover, the coaxial electrospinning approach works perfectly with low-molar mass bent core liquid crystals because they are contained inside the polymer sheath, thereby greatly expanding the pool of available and suitable materials compared to when the bent-core liquid crystal itself must be elastomeric.

\section{APPLICATION POTENTIAL}

The overview so far has brought us to a point where it should be apparent that there is a substantial potential for application of liquid crystal-containing electrospun fibers in a variety of innovative device and materials concepts. As it offers a high degree of flexibility and breathability, a functional and responsive electrospun mat is clearly of interest for wearable devices. Also on a single-fiber level, the composites can be applied, for instance, in fiber-incorporated optical nanodevices. Ultimately, it is largely our imagination that sets the limits of what can be done, certainly extending well-beyond the scope of this perspective. To give a taste of what might be expected, we end the article by discussing some plausible future application contexts for these new advanced polymer composites.

\section{Wearable Technology}

Today's enormous progress in electronics miniaturization has made us fully integrated with advanced technology. Smart phones and tablet computers are frequently our closest companions, and we have in fact reached the boundary where we experience the mismatch between the rigidity of flat hard devices and the flexibility and complex shapes of our own bodies. The next breakthrough is bound to be the crossing of this boundary, ${ }^{38,39}$ with flexible, stretchable, and preferably wearable devices replacing those of today. Wearable technology will enable entirely new devices and it will have a profound impact on the way we communicate, work, spend our leisure time, so forth. ${ }^{39}$

The interest in this development is rapidly increasing around the world, current key foci being on sensors, actuators, communication systems, and energy sources that can be integrated into our clothes. In addition, high-performance devices developed for wearable technology can find widespread usage also in other contexts due to their small size, low weight, and flexibility. Most strategies rely on surface decoration of traditional textiles, for example, by "dying" fibers with nanoparticles ${ }^{40}$ or attaching a rubber film that carries the active components. ${ }^{38}$ These approaches are close at hand but they have drawbacks.
Detachment from the carrier textile is a considerable risk and any solution based on solid state components may fail fatally when exposed to the considerable stretching, compression, and flexing occurring while wearing and handling a garment. Rubber sheets, moreover, impair breathability. The development of new materials for the emerging category of wearable high-tech devices is still in its infancy, not nearly matching the pace with which the interest in this new product concept is rising.

A more rewarding approach could for many purposes be to make the textiles themselves smart, giving them new functionality for instance by including a responsive material like a liquid crystal in the fiber core. So far, this has been done only within the limited scope of phase-change materials, yielding fibers with enhanced thermal insulation capacity, ${ }^{41}$ but functionalization with more advanced liquids would certainly open up many more possibilities. This is an applied area where liquid crystal-functionalized electrospun fibers can have a strong impact. They will not typically replace components in wearable electronic devices but they may in some cases provide nonelectronic alternatives and in other cases they will make entirely new devices possible. For instance, using cholesteric liquid crystals in the fibers (low molar mass or elastomeric), we can contemplate clothing that changes color in response to temperature change, stretching or pressure, or as a reaction to specific compounds in the surrounding atmosphere, be it air or water. Here, we would typically aim at spinning rather thick fibers because a core of at least a few $\mu \mathrm{m}$ is needed to get the full color reflection from the cholesteric. Moreover, the color can be strongly obscured by scattering due to refractive index mismatch between core and sheath, hence also the optical properties of core and sheath need to be tailored for achieving strong response. Adding concepts for gas or liquid monitoring using non-chiral liquid crystals, ${ }^{42}$ for which the latter two restrictions typically do not apply, multiple approaches to realizing autonomous garment-integrated environmental and biosensors can be considered. With flexoelectric liquid crystals and flexible electrodes in the fibers, our clothing may be turned into electricity generators that power small wearable electronic devices. Some of these possibilities are discussed in more detail below.

In terms of textiles, a difficulty with electrospun fiber mats is that it is challenging to spin them into yarns that can then be woven into a high-grade fabric. Although some progress has been made recently on this front, ${ }^{43}$ it may often be necessary to settle for nonwoven mats, which can have inferior properties to woven textiles. However, a patch of electrospun nonwoven textile can be laminated between regular textiles for easy integration ${ }^{44}$ or it can be attached on top of a single piece of carrier fabric. For many purposes, this would be a fully adequate solution. In fact, because the achievement of washability may be extremely challenging for many of the types of liquid crystal-functionalized fiber discussed here, it may be convenient to introduce these as disposable components which can be exchanged or at least removed from the carrier textile during washing. 


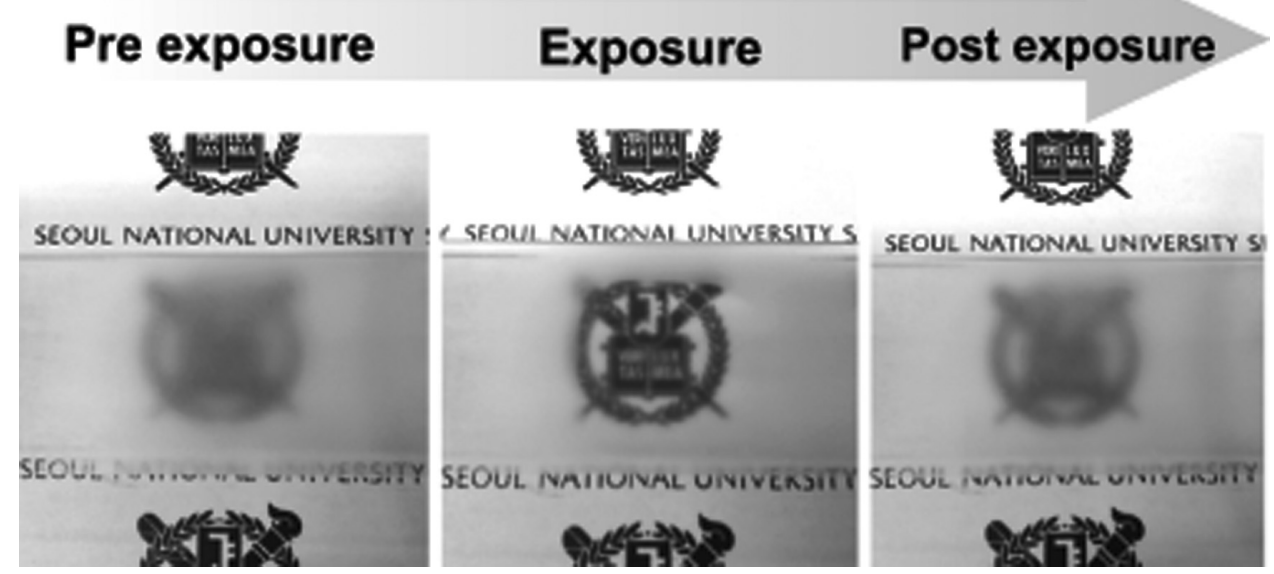

FIGURE 8 Example of a simple gas sensor made from a liquid crystal-functionalized electrospun fiber mat. The core is 5 CB, developing a nematic phase at room temperature in standard atmosphere, and the sheath is a composite of $\mathrm{PVP}_{\text {and }} \mathrm{TiO}_{2}$. In this state, the mat scatters light strongly (left). On exposure to toluene (center), the 5 CB turns isotropic, changing its optical properties such that sheath and core become almost index matched, greatly increasing the mat transparency. After stopping the toluene exposure, the core returns to the nematic state and the mat becomes scattering again (right).

\section{Liquid Crystal-Functionalized Fiber Mats Acting as Sensors}

The afore mentioned concept for using liquid crystals in gas sensing is highly attractive to combine with electrospinning. The polymer sheath provides an excellent solution for containment of the liquid crystal, protecting it from being scratched or removed due to contact with other solid materials, yet the high surface-to-volume ratio of the fibers makes the liquid crystal accessible to the vapor analytes it should detect. The small cross section of the core and the cylindrical symmetry of the interface with the surrounding atmosphere also ensures a distinct response from the liquid crystal. The radial concentration gradient of a gaseous analyte can be expected to be very small in such a thin core, whereas a bulk liquid crystal sample, typically supported on a solid substrate along half of its bounding area that limits the access to the analyte, will give a complex response at least at the beginning of the exposure to the analyte. There will then be a superposition of the response at the surface, where the analyte may have triggered a strong structural change, and that of the bulk, not yet exposed to the analyte to any considerable degree. In a fiber-based device, this blurring of the response should be minimized.

By ensuring that the sheath is spun as thin as possible and by using a polymer through which the analyte can diffuse relatively easily, a rapid response can be expected. This may be further enhanced if the sheath is given a porous morphology, following one of the procedures discussed above, ${ }^{29,30}$ as this would promote the diffusion of analyte toward the liquid crystal. However, large pores extending all the way to the interface with the liquid crystal may pose problems with capillary forces sucking it out from the central channel. This would give rise to a much more complex and largely random arrangement of $\mathbf{n}$, which at least in case of a response based on color change of a cholesteric would be a disadvantage. For scattering-based devices like the one described below, this may instead be beneficial. Compared to polymer-dispersed liquid crystals (PDLCs) an important advantage with the fibers is that the distance between liquid crystal and surrounding atmosphere is constant, defined by the sheath thickness, whereas the droplets in PDLCs are randomly distributed, many being deeply embedded in the polymer and even surrounded by other droplets. Obviously, the desire to spin the fibers with a thin sheath must be weighed against the risk of losing mechanical stability and sturdiness of the fibers. This may be supported by (partial) polymerization also within the liquid crystal core channel, as long as this does not compromise its response.

Specificity of the response can be ensured by following for example, the approach of Han et al., ${ }^{32}$ where the liquid crystal is doped with molecules tailor-designed to respond to the analyte alone. An interesting alternative may be to incorporate an analyte-sensitive surfactant, designed to concentrate at the interface between polymer sheath and liquid crystal core. If the presence of the analyte changes the surfactant conformation or in other ways affects its interaction with the liquid crystal, an alignment change of the liquid crystal phase can be expected, which will give rise to a change in optical properties that can easily be detected by eye, for example, via a change between transparent and scattering states.

A simple example of a scattering-based liquid crystal gas sensor mat, serving to prove the concept, is shown in Figure 8. The mat consists of fibers with $\mathrm{PVP}^{-T i O}{ }_{2}$ composite sheath containing a 4-pentylcyanobiphenyl (5CB) core. In air at room temperature, the $5 \mathrm{CB}$ is in a nematic state, giving it a refractive index sufficiently different from that of the sheath that the mat scatters light strongly (left photo). When exposed to toluene vapor, the 5CB in the fibers turns isotropic, leading to better index matching with the sheath such that the mat now becomes essentially transparent, revealing the logo 
placed below the mat (center photo). On stopping the toluene exposure, the core returns to the nematic state, rendering the mat scattering again (right photo). The sensing function is thus fully reversible and it is quite fast: the full transparency change of the mat takes place on the order of a second. We have not yet evaluated the sensitivity of this mat on a quantitative level, but we know that it for this particular device is not yet high enough to compete with alternative sensors. However, this mat was not in any way optimized for the purpose and we are confident that with further reduction of sheath thickness, possibly coupled to a change of the polymer material and also using a spinning procedure that ensures a porous sheath morphology (as described above), we will be able to increase the sensitivity substantially. Moreover, the liquid crystal was pristine, $5 \mathrm{CB}$, thus in no way designed to give a strong response to the analyte. The response here relies on sufficient diffusion of toluene throughout the core that the nematic phase is destabilized, a process that requires a much higher concentration of analyte than what is expected when specific receptor molecules are included.

Apart from the low footprint, low weight, low production cost, high degree of flexibility, breathability, and potentially high sensitivity of a sensor made from liquid crystal-functionalized electrospun fibers, the fully reversible operation without power supply, at room temperature, and the simple detection by eye makes this sensor/detector concept extremely attractive in diverse contexts. Although there are several practical issues to address before this type of sensor can be brought into a product, there are very few (if any) alternative sensor technologies that can offer all the listed advantages. One can easily imagine many areas where electrospun liquid crystalbased sensors can find use. The most obvious would be in clothing, for example, as patches incorporated in uniforms of factory workers or soldiers, warning them for hazardous gas leakage or the presence of biological or chemical warfare substances in the environment. Another area where they may find use is in the packaging industry as they could function as low-cost disposable sensors for example, for monitoring food quality.

\section{Actuators}

The ability of liquid crystal elastomers to change their macroscopic shape at a transition between nematic and isotropic states can certainly be used also in electrospun fibers. Although this may not have been observed in the study of Krause et al., ${ }^{20}$ it was then most likely due to practical issues with the sample preparation, as discussed above, nonfundamental issues that can be resolved in various ways. First, the fibers should preferably be spun as free-hanging mats and if possible with uniform fiber orientation. If this is difficult to achieve with the liquid crystal polymer itself, for instance because its limited molar mass renders the spinning jet unstable, the liquid crystal polymer could be spun as the core inside a supporting polymer sheath of for example, PVP. By dissolving the PVP after crosslinking the liquid crystal in the core into an elastomer, a mat that is capable of showing strong actuation should be achievable. If the sheath is itself elastomeric, for example, made out of polydimethylsiloxane or another suitable polymer that can be crosslinked during or after spinning, the actuation may be possible even without dissolving the sheath. Another possibility, finally, may be to mix in a small amount of high molar mass nonliquid crystalline polymer, sufficient to stabilize the spinning but not so much as to drastically reduce the actuation.

A requirement for efficient actuation is a monodomain sample, that is, that the director is uniformly aligned in the spinning process, but this should pose no problem as Krause et al. ${ }^{20}$ confirmed an excellent degree of director alignment throughout the fibers. By incorporating azobenzene dyes in the mixture, the transition to the isotropic state can be induced not only by temperature but also by UV light exposure. This considerably broadens the application potential of actuating mats. One could for instance consider the production of textiles that change shape in response to too long exposure to the sun, potentially useful to include in beach gear. Apart from the possible applications of actuating mats, they pose some interesting questions of more fundamental research interests, for example, which types of motion can be induced by combining different types of liquid crystal elastomers in the mats. Dynamic wrinkling following different patterns may be expected, and light- or temperature-induced directed curling or folding should be possible by smart design of the fibers.

\section{Switchable and Tunable Photonic Crystals and Fiber-Integrated Lasers}

Because a dye-doped short-pitch cholesteric liquid crystal can function as a tunable laser and as the same liquid crystal can be electrospun inside fibers, we should be able to achieve lasing also from the electrospun cholesteric-functionalized fibers. If the mat is exposed to an appropriate pumping laser, the output laser signal should then be detected radially around all fibers due to the cylindrical director configuration, generating a quite interesting new optical device. The mat could be used for instance as a tensile or pressure sensor, because stretching or compression of the fiber mat should change the lasing wavelength just like in the works of Finkelmann et al. ${ }^{11}$ and Seeboth et al., ${ }^{33}$ respectively.

But even more interesting would probably be to spin shortpitch chiral smectic-C (SmC*) liquid crystals doped with an appropriate dye inside the fibers. Also these phases provide the selective reflection required for mirrorless lasing but the geometry is different, the bandgap appearing for light propagation along the smectic layer normal. Because the smectic layers in the fibers develop perpendicular to the fibers, ${ }^{17}$ the direction of light amplification would thus be along the fibers. By spinning the fibers with a sheath polymer with lower refractive index than that of the liquid crystal at the core-sheath boundary, an optical fiber with integrated tunable lasing capacity could thus be electrospun. By polymerizing the SmC* phase, a stretching-induced bandgap tuning should be possible. Moreover, as a SmC* phase has a spontaneous polarization in the smectic layer plane that is geometrically coupled to $\mathbf{n}$, the phase responds sensitively to transverse electric 
fields. This allows electric tunability and switchability, as has been demonstrated for lasing from regular flat samples. ${ }^{45}$

\section{CONCLUDING REMARKS}

We hope that we, with this condensed overview of current trends and sketches of future development possibilities, have been able to demonstrate that electrospinning of liquid crystal-functionalized polymer fibers is an exciting research field with tremendous potential, both in terms of applications and fundamental research. The combination with more advanced coaxial spinning geometries and better optimized sheaths, as well as with conductive components like liquid metal or CNT-doped polymers, can take the field to new levels. The purpose of this perspective article is to outline some possible directions along which this young research field may develop. Our group is currently working along the lines described above and we expect to be able to show several exciting new results involving liquid crystal electrospinning in the near future. If our paper additionally can stimulate further efforts along original directions by other researchers, this would make us all the more happy. We are confident that the future for liquid crystals in electrospun fibers is bright and exciting, with plenty of room for new players and research thrusts along diverse directions.

\section{ACKNOWLEDGMENTS}

Financial support from the National Research Foundation of Korea (NRF), grant nr. 2011-0012184, and Seoul National University, Korea, grant nr. 490-20100021, is gratefully acknowledged.

\section{REFERENCES AND NOTES}

1 D. H. Reneker, A. L. Yarin, Polymer 2008, 49, 2387-2425.

2 A. Greiner, J. H. Wendorff, Angew. Chem. (Int. Ed.) 2007, 46, 5670-5703.

3 D. Li, Y. Xia, Adv. Mater. 2004, 16, 1151-1170.

4 A. L. Yarin, Polym. Advan. Technol. 2011, 22, 310-317.

5 H. Y. Chen, N. Wang, J. C. Di, Y. Zhao, Y. L. Song, L. Jiang, Langmuir 2010, 26, 11291-11296.

6 N. Wang, H. Y. Chen, L. Lin, Y. Zhao, X. Y. Cao, Y. L. Song, L. Jiang, Macromol. Rapid. Comm. 2010, 31, 1622-1627.

7 Y. Zhao, X. Y. Cao, L. Jiang, J. Am. Chem. Soc. 2007, 129, 764-765.

8 P.-G. de Gennes J. Prost, The Physics of Liquid Crystals, Clarendon Press: Oxford, UK, 1993.

9 J. P. F. Lagerwall G. Scalia, Current Appl. Phys. 2012, 12, 1387-1412.

10 H. Coles, S. Morris, Nat. Photon 2010, 4, 676-685.

11 H. Finkelmann, S. T. Kim, A. Munoz, P. Palffy-Muhoray, B. Taheri, Adv. Mater. 2001, 13, 1069-1072.

12 C. Ohm, M. Brehmer, R. Zentel, Adv. Mater. 2010, 22, 33663387.
13 M. Warner, E. M. Terentjev, Liquid crystal elastomers, Oxford University Press: USA, 2007.

14 E. Enz, J. Lagerwall, J. Mater. Chem. 2010, 20, 6866-6872.

15 J. P. F. Lagerwall, D. D. Parghi, D. Krüerke, F. Gouda, P. Jägemalm, Liq. Cryst. 2002, 29, 163-178.

16 J. P. F. Lagerwall, J. T. McCann, E. Formo, G. Scalia, Y. Xia, Chem. Commun. 2008, 42, 5420-5422.

17 E. Enz, U. Baumeister, J. Lagerwall, Beilstein J. Org. Chem. 2009, 5, DOI: 10.3762/bjoc.5.58.

18 E. A. Buyuktanir, M. W. Frey, J. L. West, Polymer 2010, 51, 4823-4830.

19 K. Nakashima, K. Tsuboi, H. Matsumoto, R. Ishige, M. Tokita, J. Watanabe, A. Tanioka, Macromol. Rapid Commun. 2010, 31, $1641-1645$.

20 S. Krause, R. Dersch, J. H. Wendorff, H. Finkelmann, Macromol. Rapid Commun. 2007, 28, 2062-2068.

21 C. Ohm, M. Morys, F. R. Forst, L. Braun, A. Eremin, C. Serra, R. Stannarius, R. Zentel, Soft Matter 2011, 7, 3730-3734.

22 S. Dölle, B. D. Lechner, J. H. Park, S. Schymura, J. P. F. Lagerwall, G. Scalia, Angew. Chem. (Int. Ed.) 2012, 51, 32543257.

23 T. Y. Liu, P. Sen, C. J. C. J. Kim, J. Microelectromech. Syst. 2012, 21, 443-450.

24 H. Yang, C. R. Lightner, L. Dong, ACS Nano 2012, 6, 622628.

25 G. Kwak, G. H. Lee, S. H. Shim, K. B. Yoon, Macromol. Rapid Commun. 2008, 29, 815-820.

26 C. Tang, C. D. Saquing, J. R. Harding, S. A. Khan, Macromolecules 2010, 43, 630-637.

27 Q. Gao, J. Takizawa, M. Kimura, Polymer 2013, 54, 120-126.

28 A. Bianco, G. Iardino, A. Manuelli, C. Bertarelli, G. Zerbi, ChemPhysChem 2007, 8, 510-514.

29 C. L. Casper, J. S. Stephens, N. G. Tassi, D. B. Chase, J. F. Rabolt, Macromolecules 2004, 37, 573-578.

30 J. T. McCann, M. Marquez, Y. N. Xia, J. Am. Chem. Soc. 2006, 128, 1436-1437.

31 F. L. Dickert, A. Haunschild, P. Hofmann, Fresenius J. Anal. Chem. 1994, 350, 577-581.

32 Y. Han, K. Pacheco, C. W. M. Bastiaansen, D. J. Broer, R. P. Sijbesma, J. Am. Chem. Soc. 2010, 132, 2961-2967.

33 A. Seeboth, D. Loetzsch, R. Ruhmann, Am. J. Mater. Sci. 2011, 1, 139-142.

34 J. S. Patel, R. B. Meyer, Phys. Rev. Lett. 1987, 58, 15381540.

35 P. Rudquist, L. Komitov, S. T. Lagerwall, Ferroelectrics 1998, 213, 53-62.

36 J. Harden, B. Mbanga, N. Eber, K. Fodor-Csorba, S. Sprunt, J. T. Gleeson, A. Jakli, Phys. Rev. Lett. 2006, 97, ARTN 157802.

37 J. Harden, M. Chambers, R. Verduzco, P. Luchette, J. T. Gleeson, S. Sprunt, A. Jakli, Appl. Phys. Lett. 2010, 96, 102907. 
38 J. A. Rogers, T. Someya, Y. Huang, Science 2010, 327, 1603 1607.

$39 \mathrm{~S}$. Seymour, Fashionable technology, the intersection of design, fashion, and technology, SpringerWien: NewYork, 2008.

40 A. G. Avila, J. P. Hinestroza, Nat. Nanotechnol. 2008, 3, 458-459.

41 J. T. McCann, M. Marquez, Y. N. Xia, Nano. Lett. 2006, 6, 2868-2872.
42 I.-H. Lin, D. S. Miller, P. J. Bertics, C. J. Murphy, J. J. de Pablo, N. L. Abbott, Science 2011, 332, 1297-1300.

43 W. E. Teo, S. Ramakrishna, Compos. Sci. Technol. 2009, 69, 1804-1817.

44 S. Lee, S. K. Obendorf, J. Appl. Polym. Sci. 2006, 102, 34303437.

45 M. Ozaki, M. Kasano, T. Kitasho, D. Ganzke, W. Haase, K. Yoshino, Adv. Mater. 2003, 15, 974-977. 